\title{
Experience the World with Archetypal Symbols: A New Form of Aesthetics
}

\author{
Huang-Ming Chang ${ }^{1,2}$, Leonid Ivonin ${ }^{1,2}$, Marta Diaz $^{1}$, Andreu Catala ${ }^{1}$, \\ Wei Chen ${ }^{2}$, and Matthias Rauterberg ${ }^{2}$ \\ ${ }^{1}$ CETpD Research Center, Universitat Politècnica de Catalunya, Barcelona, Spain \\ \{marta.diaz, andreu.catala\}@upc.edu \\ ${ }^{2}$ Dept. Industrial Design, Eindhoven University of Technology, The Netherlands \\ \{h.m.chang, 1.ivonin, w. chen, g.w.m.rauterberg\}@tue.nl
}

\begin{abstract}
According to the theories of symbolic interactionism, phenomenology of perception and archetypes, we argue that symbols play the key role in translating the information from the physical world to the human experience, and archetypes are the universal knowledge of cognition that generates the background of human experience (the life-world). Therefore, we propose a conceptual framework that depicts how people experience the world with symbols, and how archetypes relate the deepest level of human experience. This framework indicates a new direction of research on memory and emotion, and also suggests that archetypal symbolism can be a new resource of aesthetic experience design.
\end{abstract}

Keywords: Human Experience, Symbols, Phenomenology, Archetypes.

\section{Introduction}

Interaction involves a series of expression and interpretation between us as human beings and the world around us. Speaking of aesthetics in interaction, what intrigues us more is the very moment when meaning emerges while human beings are experiencing the world. Through experiencing the world, human beings then know how to appreciate the beauty of interaction. Symbolic Interactionism [1] is a sociological theory that aims at analyzing the patterns of communication, interpretation and adjustment between people. This theory provides a framework for understanding how people interact with each other through the meanings of symbols. A fundamental premise is that people do not directly react to the ontological-existing reality, but respond to their understanding of this reality. Contrary to the traditional view of human in the machine paradigm, humans act toward things on the basis of the meanings that they ascribe to these things [2]. People interact with each other by interpreting each other's actions instead of merely reacting to each other's actions. Their response is not made directly to the actions of one another, but instead is based on the meaning that they attach to such actions. That is, each action, object, or event has its own symbolic meaning to be revealed. It is symbols that bridge the gap between the physical 
reality and what humans perceive, feel, and understand as a reality. While the hypothesis is made, a further question is raised: how are symbols created? Symbolic interactionism assumes that symbolic meaning emerges while interaction is happening between people within the same social context. On the other hand, symbolic meaning is in turn given by the social context where the interaction is situated. This forms a reciprocal causation relationship between the symbolic meaning and the interaction.

The hypothesis of symbolic interactionism shared similarity with some discussions about human experience in cognitive science [3]. Human experience has been a tough topic for researchers. The tradition of behavioral science sees the physical world as the true reality, and even to the extreme, argues that psychological phenomena are just illusions [4]. Therefore it only takes into account how human bodies function in response to external stimuli, and rejects the existence of human mind. Nevertheless, while some researchers try to describe the physical world as an independent body of knowledge, phenomenologist Husserl [5] argues that human can never rid themselves of the world and observe it objectively. The world, as a phenomenon, only emerges while we are living within it. Therefore, he proposes the concept of 'life-world', saying that the world is a grand theater of objects variously arranged in space and time relative to perceiving subjects. The life-world is already and always there, and can be thought of as the background for all human experiences. All our personal experiences can thus be built upon this background through living within it. In this sense, the world we are talking about is never ontologically-objective, but always ontologicallysubjective to the life-world we all share. It is argued that this life-world is considered as the real reality that researchers should focus. What we need to further understand is how personal subjective experience is built upon this consensual reality.

It seems that behaviorism and phenomenology hold completely opposite positions on their understanding about human experience. Fortunately, this dilemma was resolved when 'phenomenology of perception' was proposed [6]. Inherited from Husserl's concept of 'life-world,' Merleau-Ponty also believes that the physical world and what we experience as the world are different but inseparable realities. However, different from the above dualistic point of view, he further claims that the perception (or the sensation) is the channel that communicates the physical world and the experienced world. That is, he acknowledges that both physical world and the life-world exist as realities. For example, when you open your eyes, your perception is actively automated to senses the red color or any other attributes of the physical world. When you shut your eyes, you can still embody the redness or any other qualities - as part of the lived-world-in your mind. In this sense, psychology and physiology are no longer two paralleled science, but two accounts of human experience [6]. Based on this argument, a further question to answer is that: what are the media that transmit the information between the physical world and the life-world?

This question has been long annoying researchers since they tried to define psychological phenomena. Since we have acknowledged that these psychological phenomena are real, what we need to answer is that how these phenomena become real [4]? Psychology thus comes into play. Some cognitive scientists hold a similar opinion with symbolic interactionists. They argue that symbols, as the media that bear 
meaning, flow through the channel of perception between the ontologically-objective world and ontologically-subjective human experience [3].

\section{Experience the World with Symbols}

What are symbols? Do symbols equal to signs? A Sign is a representation of one concrete concept that implies a direct connection between itself and the concept it refers to. What it means 'direct connection' is that this connection to the extreme leads to a causal relationship. For example, thunders are usually known as the sign of a storm due to the fact that thunders always come with storms. In contrast, symbols are used to signify things without rational correlations, such as a flag is a symbol of a country. It is further argued that sign can only be used to refer to the known things, whereas symbols indicate something that is still unknown, or ideas that cannot be precisely depicted [7], e.g. peace, love, and culture. In essence, symbols itself are ontologically objective, and bear no psychological meaning. Their meanings emerge only when one's life-world is being lived. By saying this means that the meaning of symbols is ontologically subjective to human knowledge. Therefore, symbols can be in any kind of forms or values of anything in the physical world depending on how we approach the physical world to reveal our life-world [8]. In other words, the meaning of symbols would vary based on which layers of knowledge are adopted to support the experiencing procedure. Opposite to explicit knowledge that needs to be acquired by conscious learning and repetitive remembering, Sperber [9] argues that symbolism is a kind of tacit knowledge, an autonomous cognitive mechanism that, alongside the perceptual and conceptual mechanisms, participates in the construction of knowledge and in the functioning of memory. However, different from semiology, symbolic interpretation is not a matter of decoding, but an improvisation that rests on an implicit knowledge and obeys unconscious rules. He further propose a hypothesis that the basic principles of the symbolic mechanism are not induced from experience but are, on the contrary, part of the innate mental equipment that makes experience possible.

In the traditional psychological concept, especially behaviorism, humans are usually understood with a "stimulus-response" process. However, a gap emerges between the physical matters and psychological states. In research of emotion, appraisal theories [10] provides an fine argument to bridge this gap. While one is being chased by a tiger, it is not the 'physical' tiger that causes the fear, but the appraisal of this situation-being chased by a tiger-triggers this emotion of fear. This theory thus calls into the question: what provides the reference for the process of appraisal? Barrett [4] claims that humans share a kind of 'category knowledge' in their cognition process to interpret the ontologically-subjective meaning of ontologically-objective events. This knowledge enables the psychological phenomena to link the body to the world to create meanings. She further argues that the society can be the source of the knowledge. The society, by the definition of symbolic interactionism [1], is a reality that is embodied through interaction among the people within it. On the other hand, the society in turn provides the symbolic meaning of everything within its social context. Symbolic meaning is seen as a dynamic phenomenon, being constructed while 
interaction occurs, and in turn, grounding the basic understanding among people within it. It could also be understood as a pre-understanding of the physical world. Extended from this pre-understanding by living within it, humans create their new understanding, which in turn becomes the pre-understanding of the society. This loop is so-called hermeneutic circle [11]. Interestingly, not only symbolic interactionists but also some psychologist support the same idea that the society should be one of the sources of symbolic meaning [4]. It is obvious that people in the same society to some extent share the same languages, value system, and even ways of thinking. This knowledge is not always explicitly given through education, but more often is gained implicitly through living within it.

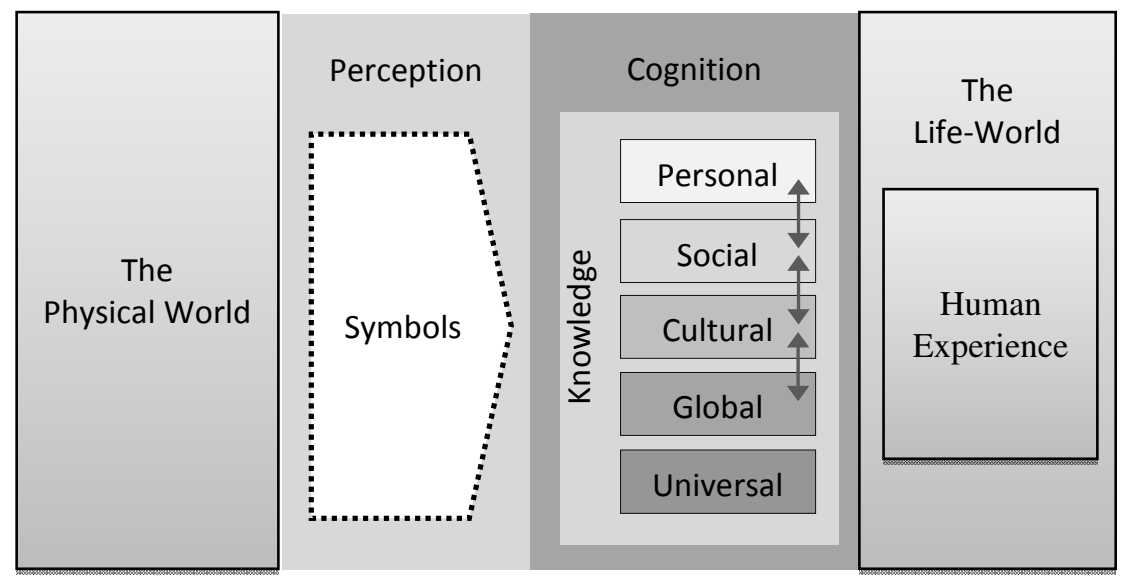

Fig. 1. The framework of how human experience is built with symbols

Beyond the society, we argue that there are many levels of knowledge that supports our building process of the life-world (see figure 1). These five levels of knowledge are personal, social, cultural, global, and universal. These first four levels of knowledge are neither static nor independent, but in a dynamic circulation. Information flows through different levels, influencing their next level of knowledge simultaneously. Top four levels of knowledge change differently with time [12] while the deepest level of knowledge remains consistent across time and space, as we call it universal knowledge of symbols.

\section{Archetypal Symbolism}

Psychologist Jung [7] reveals insights about unconsciousness in a wider sense throughout analyzing myths and fairytales from numerous cultures. He proposed the concept of 'collective unconsciousness' [13], arguing that besides the personal psyche (includes both conscious and unconscious minds), there exists a deeper level of unconscious mind, which contains some contents and modes of behavior that are identical in all individuals over time and space. The collective unconsciousness thus 
constitutes a common psychic substrate of a universal nature which is present in every one of us. This theory shares a similar idea with the Hindu view of reality [14] (see Figure 2). Egos of people are like separated islands above the water, as people see each other as independent individuals in the physical world. Our conscious thinking makes us believe that we are separate entities who are floating freely above the water. However, people can hardly notice the unconscious part of their mind under the surface of water. Furthermore, people are unaware that they are connected to each other by means of the ocean floor beneath the water. This is what Jung claimed that our personal unconsciousness rests upon a deeper layer, the collective unconsciousness, which is not a personal acquisition but is inborn as the foundation of the psyche [13].

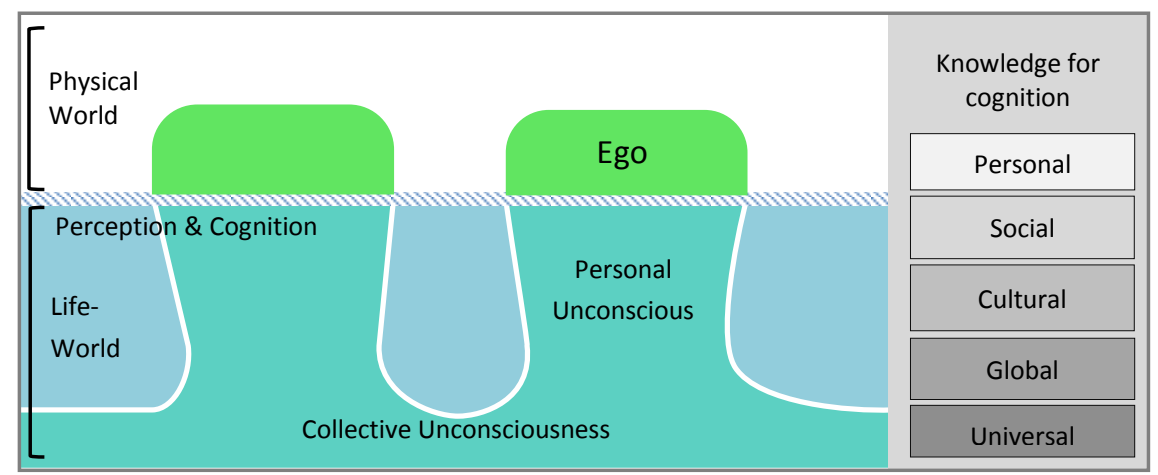

Fig. 2. A metaphorical mapping of the levels of knowledge and Hindu view of reality (adapted from Boeree [14])

Applying the same analogy with Jung's theory to our framework of human experience, a more thorough explanation can be drawn (see figure 2): the world above the water represents the physical world while the world under water refers to the lifeworld [5]. Human perception and cognition therefore appears to be the fine surface that connects between the air (the physical world) and the sea (the life-world). The water that surrounds us-as the society and the culture we live in-shapes our personal unconsciousness in an implicit way [15]. Besides the upper four levels of knowledge, the level of universal knowledge at the bottom links to the collective unconsciousness, which stands as the fundamental sea floor that we all connect with.

Continuing with the theory of the collective unconsciousness, Jung further developed the concept of archetypes [7]. Archetypes are defined as the components of the collective unconsciousness, which is an inborn tendency that cannot be consciously acquired to experience things in a certain way [13]. As the receptive fields of retina are not consciously perceived, but forms visual perception, archetypes, likened to another model of human, are psychic structures of a primordial origin, which are mostly inaccessible to consciousness, but determine the structure of our psyche [16]. Archetypes are very close analogies to instincts because the latter are impersonal, inherited traits that present and motivate human behavior long before any consciousness develops. Jung described archetypes as an unconscious psychic impulse, like 
instincts influence people as physical impulse toward actions [17]. A more extreme analogy would be describing archetypes as the structure of the psyche, which is similar to organs of the physical body [18]. By the same token, if our body functions as the 'hardware' of our perception, i.e. our physical organs, archetypes play as the role of 'software' that define our patterns of thoughts, emotions, and behaviors. Hence, symbols appear to be data, flowing between the physical world and the life-world.

Although archetypes are embedded in the deepest level in human unconsciousness, Jung found that archetypes are embodied as ancient motifs and predispositions to patterns of behavior that manifest symbolically as archetypal images in dreams, art, or cultural forms [7]. His followers continue this direction, collect archetypal symbolic contents all over the world, and analyze their symbolic meanings from both archeological and psychological perspectives [19]. These data provide an abundant resource for not only research but also design of human experience.

\section{Implication for Research}

Human experience (the life-world) is an ontologically-subjective phenomenon, which we cannot direct observe. To unfold this black box, the only option is to analyze the representation of these mental phenomena. For this purpose, it is suggested to seek the paradigms in psychology [4]. Since humans' unconsciousness is hardly accessible by their consciousness, it is unclear if some psychological representations are triggered by one's conscious thinking, unconscious thinking, or even both. For example, if we simply apply the 'memory-recall tasks' method: directly ask subjects to recall the semantic meaning of a symbol according to their own understanding. This approach is problematic for two reasons. First, semantic expression has limits in its nature, so that it might not be able to reflect complete symbolic meanings. As Jung states, symbolic meanings are abstract concepts that cannot be precisely described [7]. Second, memory-recall task is a conscious inference process for explicit memories (or declarative memories) [20,21], which is supported by the personal level of knowledge instead of other knowledge that relate to unconsciousness. In contrast, implicit memories (or non-declarative memories) are unconscious and associative, which are suggested to apply association tasks in experiments [22].

Several studies have demonstrated the effectiveness of memory-association tasks in justification of the symbolic meanings for archetypal contents [23, 24]. This is also in accord with the mainstream of research into memories: the constructionist approach of memory recollection [25]. This approach regards memories as dynamic recollections instead of static records of something. Each recall task requires 'cues' that are is associated with fragments of memories of something. Those fragments of memories with strong connections with the cue would be easier to be cued. A similar paradigm is adopted in research on emotion, where researchers follow a stimuli-representation procedure. Although this procedure makes very few differences to traditional behaviorist methods, modern psychologists consider subjects' emotional responses as an indirect representation of their psychological phenomena instead of direct responses to the given stimuli. More importantly, researchers have found that emotions play the key role in 
strengthening the association between the fragment of memories and its cue [26]. This allows us to extend our framework in more details (see figure 3). In the context of our framework of human experience, some physical attributes in the physical world are identified as symbols according to the five levels of knowledge in the cognition process, and then are embodied as symbols in the life-world. Symbols function both as the cue that extracts the related memories and also the stimuli that elicit emotions out of subjects' life-world. These psychological phenomena aggregate as a new experience that emerges from the life-world. Each of new experiences represents the emergence of a new symbolic meaning. While an experience is embodied in the life-world, it is simultaneously influencing the knowledge for cognition. This reflects the concept of the hermeneutic circle [11], that humans change their way of thinking (knowledge for cognition as pre-understanding) while they are experiencing the world (human experience as understanding). The new experience again becomes memories in the life-world for future experience and recollection of memories.

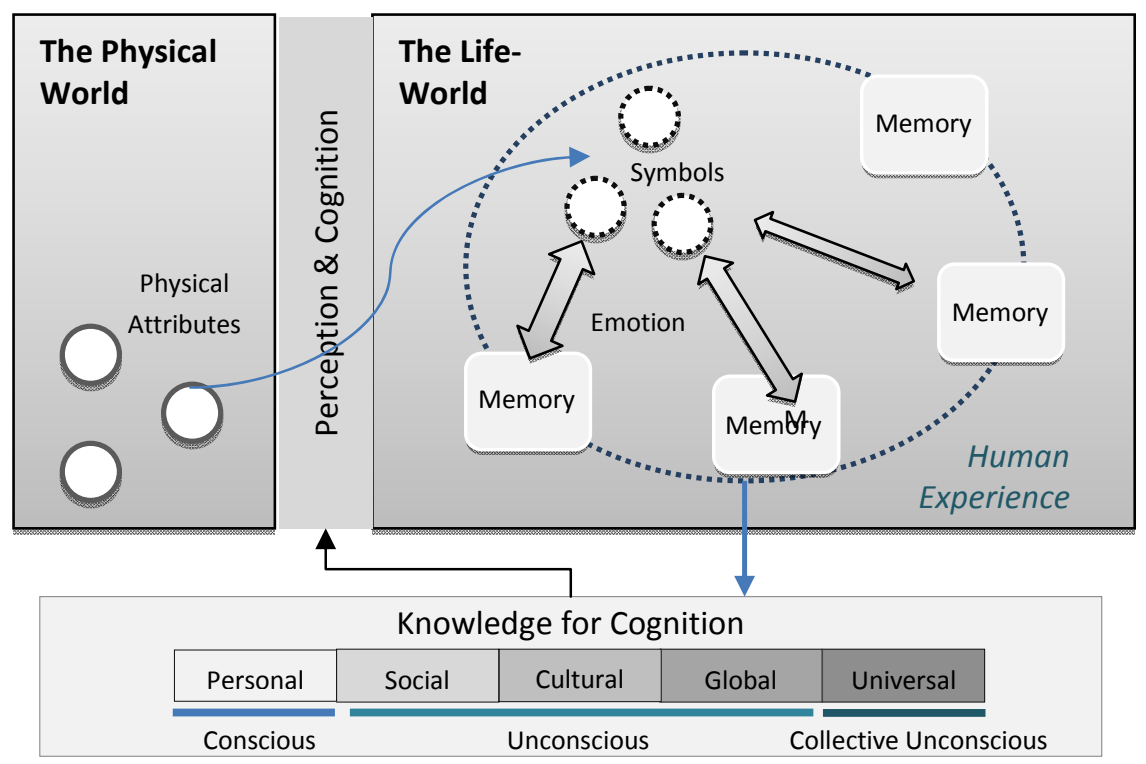

Fig. 3. Our advanced framework of how human experience is formed in the life-world

As the main focus of this framework is on the universal level of knowledge, i.e. archetypes, numbers of interesting research questions are raised for future work. First, how can we study archetypes? Since archetypes are hidden in the unconscious, the only way to study them is through their representation in different forms, such as stories, graphics, and even movies. Therefore, a starting point would be identifying archetypal contents as the materials that can be used for psychological experiments. Second, what is the correlation between archetypes and human experience? This research question actually involves two intertwined psychological phenomena: emotion and memory. Since these emotions and memories about archetype are all in the 
unconscious level, it suggests indirect approaches to capture these psychological phenomena, e.g. memory-association tasks instead of memory-recall task [23, 24], and physiological signals instead of self-reports of emotions [27].

\section{Design for Aesthetic Experience}

A more interesting topic to implication for design is how archetypal symbolic contents can be applied in design for aesthetic experience. Traditional product design mainly focuses on aesthetic experience, experience of meaning, and emotional experience in physical products [28]. With this definition, aesthetic experience relies on one's appreciation of the form and material, whereas the experience of meaning counts on the interpretation of the personal, social, cultural meanings of the products. In the end, emotional experience emerges as the overall result of the appraisal of these two experiences. However, Ross and Wensveen [29] propose another form of aesthetics: aesthetic interaction. They emphasize the importance of the social and ethical dimensions in dynamic forms of interaction. In which case, aesthetics is not bounded with physical attributes of products, and can manifest through interacting with products. What designers should take into account is the symbolic meaning of the interaction among users and products, rather than the intrinsic meaning of the physical products itself.

Physical products, in our framework, are part of the physical world, which are ontologically-objective and bear no meanings. These products then become stimuli of emotions or cues of memories, which initiate an internal process of experience and external behavior interacting with it. Apart from personal appreciation about the static qualities, e.g. form and material of products, the aesthetics experience also emerges when people are unconsciously yet actively trying to reveal the symbolic meaning of the product as part of their life-world. This perspective of product experience goes beyond the function of product, focusing on the presence of objects and the meaning of the interaction between human and product. Niedderer proposes a framework of this interaction of meaning making: mindful interaction, and names the products with the quality of meaning making as performative objects [30]. More specifically, mindfulness refers to a state of awareness or consciousness that implies one's presence of the moment, and is believed to promote psychological well-being [31]. The concept of performative objects addresses the product's consequences for human social interaction, raising awareness and reflection of oneself in the present. The ultimate goal of this framework is to enhance users' states of mindfulness through the mediating influence of products. According to our framework of human experience, mindful interaction demonstrates a kind of approach to transform physical attributes in physical world to symbols in the life-world, and with this interaction, users are guided to reflect the social phenomena behind the usage of the product. In other words, the attempt of mindful interaction is to bring the knowledge for cognition to a conscious level, allowing users to be aware of the experience that they are undergoing.

While Niedderer's framework focuses on social phenomena, we aim at leading users to a deeper level of their own experience, i.e. experience about archetypes. In this 
sense, archetypal symbolism provides us an opportunity to design a new form of aesthetic experience in a unconscious level. One typical expression of the experience about archetypal symbols might be Campbell's concept of the hero's journey [32]. Based on Jung's theory of archetypes, he identifies the basic structure of archetypal experience in all myths from different cultures in human history. This also sheds light on some study in user experience about using new products [33]. Metaphorical speaking, the storyline of hero's journey implements the construction of the life-world built by archetypal symbols. It seems promising to apply the storyline of the hero's journey as a new approach to achieve mindful interaction that leads users to achieve archetypal experiences.

\section{Conclusion}

Based on the theories of symbolic interactionism, phenomenology of perception, and archetypes, we introduce a new framework describing how human experience the world with symbols. In this framework, archetypes are the components of the deepest level of knowledge for cognition, the collective unconsciousness, which defines the basic structure of the life-world. This concept indicates a new direction of research on human experience. Furthermore, we suggest that archetypal symbolism can be a new resource of aesthetic experience design.

Acknowledgements. This work was supported in part by the Erasmus Mundus Joint Doctorate in Interactive and Cognitive Environments (ICE), which is funded by the EACEA Agency of the European Commission under EMJD ICE FPA n 2010-0012.

\section{References}

1. Manis, J.G., Meltzer, B.N.: Symbolic Interaction: A Reader in Social Psychology. Allyn and Bacon, Inc. (1978)

2. Kohler, A.: To think human out of the machine paradigm: Homo Ex Machina. Integrative Psychological \& Behavioral Science 44, 39-57 (2010)

3. Varela, F.J., Thompson, E.T., Rosch, E.: The Embodied Mind: Cognitive Science and Human Experience. MIT Press (1992)

4. Barrett, L.F.: Emotions are real. Emotion 12, 413-429 (2012)

5. Husserl, E.: The Crisis of European Sciences and Transcendental Phenomenology: An Introduction to Phenomenological Philosophy. Northwestern University Press (1970)

6. Merleau-Ponty, M.: Phenomenology of Perception. Routledge (2002)

7. Jung, C.G.: Man and His Symbols. Doubleday, Garden City (1964)

8. White, L.A.: The symbol: The origin and basis of human behavior. Philosophy of Science 7, 451-463 (1940)

9. Sperber, D.: Rethinking Symbolism. Cambridge University Press (1975)

10. Scherer, K.R.: What are emotions? And how can they be measured? Social Science Information 44, 695-729 (2005)

11. Gadamer, H.-G.: Truth and Methods. Sheed \& Ward Ltd. (1975) 
12. Kooijmans, T., Rauterberg, M.: Cultural computing and the self concept: Towards unconscious metamorphosis. In: Ma, L., Rauterberg, M., Nakatsu, R. (eds.) ICEC 2007. LNCS, vol. 4740, pp. 171-181. Springer, Heidelberg (2007)

13. Jung, C.G.: The Archetypes and the Collective Unconscious. Princeton University Press, Princeton (1981)

14. Boeree, C.G.: Carl Jung, http: / / webspace. ship. edu/cgboer/jung . html

15. Wilson, T.D.: Knowing when to ask: Introspection and the adaptive unconscious. Journal of Consciousness Studies 10(10), 131-140 (2003)

16. Perlovsky, L.: Neural dynamic logic of consciousness: The knowledge instinct. In: International Joint Conference on Neural Networks, IJCNN 2006, pp. 377-383. IEEE (2006)

17. Jung, C.: Instinct and the unconscious. The British Journal of Psychology 10, 15-23 (1919)

18. Jacobi, J.: The Psychology of C. G. Jung. Yale University Press (1973)

19. Ronnberg, A., Martin, K.: The Book of Symbols: Reflections on Archetypal Images. Taschen (2010)

20. Schacter, D.L.: Implicit memory: History and current status. Journal of Experimental Psychology: Learning, Memory, and Cognition 13, 501-518 (1987)

21. Squire, L.R.: Declarative and nondeclarative Memory: Multiple brain systems supporting learning and memory. Journal of Cognitive Neuroscience 4, 232-243 (1992)

22. Greenwald, A.G., Poehlman, T.A., Uhlmann, E.L., Banaji, M.R.: Understanding and using the Implicit Association Test: III. Meta-analysis of predictive validity. Journal of Personality and Social Psychology 97, 17-41 (2009)

23. Bradshaw, S., Storm, L.: Archetypes, symbols and the apprehension of meaning. International Journal of Jungian Studies, 1-23 (2012)

24. Rosen, D.H., Smith, S.M., Huston, H.L., Gonzalez, G.: Empirical study of associations between symbols and their meanings: Evidence of collective unconscious (archetypal) memory. Journal of Analytical Psychology 36, 211-228 (1991)

25. Van den Hoven, E., Eggen, B.: Informing augmented memory system design through autobiographical memory theory. Personal and Ubiquitous Computing 12, 433-443 (2007)

26. Szpunar, K.K., Addis, D.R., Schacter, D.L.: Memory for emotional simulations: Remembering a rosy future. Psychological Science 23, 24-29 (2012)

27. Ivonin, L., Chang, H.-M., Chen, W., Rauterberg, M.: Unconscious emotions: Quantifying and logging something we are not aware of. Personal and Ubiquitous Computing, 1-11 (2012)

28. Desmet, P., Hekkert, P.: Framework of product experience. International Journal of Design 1, 1-10 (2007)

29. Ross, P.R., Wensveen, S.A.G.: Designing behavior in interaction: Using aesthetic experience as a mechanism for design. International Journal of Design 4, 3-13 (2010)

30. Niedderer, K.: Designing mindful interaction: The category of performative object. Design Issues 23, 3-17 (2007)

31. Brown, K.: The benefits of being present: Mindfulness and its role in psychological wellbeing. Journal of Personality and Social Psychology 84, 822-848 (2003)

32. Campbell, J.: The Hero with A Thousand Faces. Princeton University Press (1973)

33. McLoone, H.E.: Product archetype of personal computers as an expression of the collective unconsciousness of people on their hero's journey. Proceedings of the Human Factors and Ergonomics Society Annual Meeting 54, 1771-1775 (2010) 